\title{
Relation between Eating Evoked by Lateral Hypothalamic Stimulation and Tail Pinch in Different Rat Strains ${ }^{1}$
}

\author{
Elliot Valenstein, Israel Lieblich, ${ }^{*}$ Rivka Dinar, ${ }^{*}$ Edna Cohen, ${ }^{*}$ \\ and Susan Bachus \\ Department of Psychology, University of Michigan, Ann Arbor, Michigan 48109, and \\ *The Hebrew University of Jerusalem
}

\begin{abstract}
The similarity between the behaviors evoked by tail pinch (TP) and electrical stimulation of the lateral hypothalamus (ESLH) has been noted by many investigators. Evidence is presented for a possible inverse relationship between the probability that ESLH would evoke eating and the readiness to eat in response to TP in six different rat populations (High and Low lines of the LC1 and LC2 Hebrew University Strains, Sprague-Dawley, and Long-Evans). Discussion of these results emphasizes differences in intensity between ESLH and TP stimulation and differences in emotionality among rat populations.
\end{abstract}

The similarities between the behaviors evoked by electrical stimulation of the lateral hypothamus (ESLH) and tail pinch have been described by several investigators (Antelman, Rowland, \& Fisher, 1976a; Rowland \& Antelman, 1975; Valenstein, 1976; Valenstein, 1977). It has been observed, for example, that both ESLH and tail pinch can evoke a range of different behaviors such as eating, licking and gnawing without any modification of the stimulus parameters. Within as yet undefined limits, the particular behavior displayed may depend on the goal objects available. In rats, tail pinch (or skin shock) and ESLH can also facilitate other behaviors such as male sexual behavior (Barfield \& Sacks, 1968; Caggiula, 1972; Caggiula \& Eibergen, 1969), aggression (Caggiula, 1972; Panksepp, 1971), and retrieval of pups (Mendelson, 1972; Phillips, Cox, Kakolewski, \& Valenstein, 1969; Szechtman, Siegel, Rosenblatt, \& Komisaruk, 1977). The most striking difference between the two stimuli is that tail pinch does not seem to induce rats to drink plain water although animals will consume liquid food and sweetened water in response to tail pinch (Marques, Fisher, Okrutny, \& Rowland, 1979; Mufson, Balagura, \& Riss, 1976).

\footnotetext{
${ }^{1}$ The authors want to express their appreciation to the United States-Israel Binational Science Foundation for support and to the Council for International Exchange of Scholars (Fulbright-Hays) and the United States-Israel Education Foundation for a fellowship to E.S.V.
} 
In addition to the variety of behaviors that have been observed during both ESLH and tail pinch, other parallel properties of the evoked behaviors have been noted. Rowland and Antelman (1976) have reported that tail pinch can force rats to overeat and become obese, as Steinbaum and Miller (1965) had demonstrated with ESLH. Moreover, both tail pinch and ESLH facilitate the recovery of aphagic rats after lateral hypothalamic ablations (Antelman, Rowland, \& Fisher, 1976b; Harrell, Raubeson, \& Balagura, 1974; Mufson et al., 1976; although see Greenspon \& Fass, 1981). Tail-pinch-induced behaviors also provide sufficient motivation for learning in rats (Koob, Fray, \& Iversen, 1976) as had been reported earlier with ESLH (Coons, Levak, \& Miller, 1965; Mendelson, 1966; Roberts \& Carey, 1965). It has been claimed that both tailpinch and ESLH-evoked eating are mediated by dopaminergic systems (Antelman \& Szechtman, 1975; Antelman, Szechtman, Chin, \& Fisher, 1975; Phillips \& Fibiger, 1973, 1976; Phillips \& Nikaido, 1975; Sahakian \& Robbins, 1977).

There is now convincing evidence that animals differ in the likelihood that they will eat or drink in response to ESLH, and this difference cannot be explained by electrode placement. For example, rats with two electrodes in different locations within the hypothalamus are more likely to eat or drink in response to stimulation via a second electrode if they had shown these behaviors when stimulated with the first electrode (Mittleman \& Valenstein, 1981; Valenstein, Cox, \& Kakolewski, 1970). Rats with moveable electrodes continue to eat and drink after the electrode position has been changed several times within the hypothalamus while other animals do not exhibit those behaviors at any electrode placement (Wise, 1971). Moreover, Bachus and Valenstein (1979) have demonstrated that following large lesions around the tip of the electrode, animals will continue to exhibit the same evoked behavior even though higher currents are required to stimulate more distant intact tissue. These studies all argue strongly for individual differences in the probability that ESLH will evoke eating and drinking. Recently, it has been shown that there are also significant strain differences in the probability of obtaining ESLH-induced eating and drinking that similarly cannot be explained by electrode placement (Mittleman \& Valenstein, 1981).

Since ESLH and tail-pinch behavior have so many common properties, it could be hypothesized that they might therefore be governed by common mechanisms. If such a relationship were to hold within every animal, then a positive correlation should also exist between groups of animals differing in these two behaviors. The existence of strains of animals that differ in the probability of displaying ESLH-induced eating provides a method for testing whether these strains also differ in tail-pinch-induced eating, without involving the possibility of "carryover effect," which could be anticipated if the same animals were tested with both types of 
stimulation. While the probability of inducing tail-pinch eating may approach $100 \%$ (Antelman \& Szechtman, 1975), we have consistently observed that animals differ significantly in readiness to display such behavior. Individual animals may start to eat almost immediately after the tail pinch is first applied. Others may require a number of trials before they eat at all and then they may eat only relatively sparingly. In our experience, some animals fail to eat even after repeated exposures to tail pinch. The present experiment, therefore, was designed to compare the relationship between the incidence of ESLH-induced eating and the readiness to display tail-pinch eating in four different strains of rats.

\section{GENERAL METHODS}

\section{Subjects}

The subjects were 371 male and female rats from four different strains. The weight of the animals ranged between 225 and $450 \mathrm{~g}$ at the start of the experiment with no significant difference in body weight between any of the strains. The strains were the Sprague-Dawley (S-D) albino rats (Holtzman Co., Madison, Wisc.), Long-Evans (L-E) hooded rats (Simonsen Co., Gilroy, Calif.), and the LC1 and LC2 albino strains from the Hebrew University Colony.

The LC1 and LC2 strains were each further subdivided into high (Hi) and Low (Lo) lines based on a selection procedure for high or low rates of lateral hypothalamic self-stimulation. Because details of the background and breeding procedure of these strains have been described elsewhere, it is sufficient here to indicate that animals from LC1 and LC2 families that had achieved self-stimulation rates above and below median scores were bred to form Hi and Lo lines, respectively (Lieblich, Cohen, \& Beiles, 1978). Animals used in the present study were from the eighth generation of selective breeding for the LC1 and from the seventh generation for LC2. Within the LC2 strains there is now a significant difference in self-stimulation rates between the $\mathrm{Hi}$ and Lo lines (Ganchrow, Lieblich, \& Cohen, 1981). The LC1 strain have a lower self-stimulation rate than the LC2 strain, and the phenotypic difference between the $\mathrm{LC1} \mathrm{Hi}$ and Lo is smaller and therefore harder to demonstrate in small samples. Recently, it has been established that female rats of both the $\mathrm{LC} 1$ and $\mathrm{LC} 2$ strains have significantly higher selfstimulation rates than do male rats (Cohen \& Lieblich, 1981). Of the eight groups (males and females in the two lines in each of the two strains), the LC2 Hi females have the highest self-stimulation rates. Other behavioral and physiological differences between the $\mathrm{Hi}$ and Lo lines of the LC1 and LC2 animals have been established (Ganchrow, Lieblich, \& Cohen, 1981; Inbal et al., 1980; Lieblich, Cohen, Marom, \& Dymshitz, 1980 ), but prior to the present experiment they had not been tested for 
either ESLH- or tail-pinch-induced eating. L-E rats display significantly more ESLH-induced eating than do the S-D animals (Mittleman \& Valenstein, 1981), but their behavior in response to tail pinch has not been compared.

\section{General Procedure}

Animals were housed in individual cages in a temperature-regulated room. Food and water were available ad libitum. Lights were on for 12 $\mathrm{hr}$ each day. Animals were given from 3 days to 1 week recovery time after surgery before any testing was initiated. In the evaluation of all statistical test results, a probability of less than .05 was used for rejecting the null hypothesis.

\section{Electrode Implantation}

Under general anaesthesia (sodium pentobarbital), bipolar electrodes were implanted stereotaxically at 3.20 to $3.50 \mathrm{~mm}$ posterior to bregma, 1.25 to $1.50 \mathrm{~mm}$ lateral to the midline, and 7.9 to $8.2 \mathrm{~mm}$ below the dura, with the skull held level between bregma and lambda. The electrodes were made from twisted stainless-steel wire $(.25 \mathrm{~mm}$ in diameter) insulated with Teflon except for the adjacent tips. The electrodes were fixed to the skull with cranioplastic cement and stainless-steel screws.

\section{ESLH-Evoked Eating Tests}

The animals that were given ESLH-evoked eating tests were placed in a $25 \times 20 \times 50-\mathrm{cm}$ testing chamber in which small, broken pieces of Purina Lab Chow pellets were scattered over the floor. Animals were allowed to habituate to the test chamber for at least $15 \mathrm{~min}$ or longer if they displayed any tendency to eat spontaneously. Stimulation consisted of $50-\mathrm{Hz}$ sine waves from a constant current source. Stimulus trains of $20-\mathrm{sec}$ duration were separated by a $15-\mathrm{sec}$ interstimulus interval. Stimulation intensity was raised in $1-\mu \mathrm{A}$ steps from $1 \mu \mathrm{A}$ rms until the animal was observed eating food or until the stimulus had reached an intensity that agitated the animal excessively. If the animal did not eat during stimulation at any intensity, the test was repeated the next day, and in the event that it still did not eat during the second test, testing was terminated and the animal was classified as ESLH negative. For animals that ate during stimulation, the current intensity judged to be optimal was repeated at least 10 times, and if the animal ate during at least $50 \%$ of the stimulus presentations, it was classified as ESLH positive. Animals that did not meet this criterion were considered ESLH negative. Using the above criteria, all animals could be classified as ESLH positive or negative. 


\section{Tail-Pinch Tests}

Following the procedure described by Koob, Fray, and Iversen (1976), animals were placed in a chamber $25 \times 20 \times 50 \mathrm{~cm}$ in which the floor was littered with broken food pellets of various sizes. After a 2-min habituation period, a paper clip (British Giant Serrated Paper Clips, 80 $\mathrm{mm}$, Ref. 0171A01) padded with electrical tape was applied approximately $3.5 \mathrm{~cm}$ from the tip of the tail. The clip was left on for $2 \mathrm{~min}$, during which time a record of whether or not the animal ate any food was recorded. This 2-min habituation period followed by 2-min paperclip test was repeated on 4 consecutive days for LC1 and LC2 rats. Each animal was given a score ranging between 0 and 4 depending on how many of the tail-pinch tests evoked eating. The same procedure was used for the $\mathrm{L}-\mathrm{E}$ and $\mathrm{S}-\mathrm{D}$ rats except that they received 10 daily tests in a chamber $65 \times 40 \times 50 \mathrm{~cm}$. The total time the S-D and L-E rats spent eating and orienting to the paper clip was also recorded.

\section{EXPERIMENT 1}

A random sample of 327 male and female rats from the $\mathrm{Hi}$ and Lo lines of the LC1 and LC2 strains were tested for either ESLH or tailpinch-evoked eating. The testing procedure has been described under Methods. Table 1 presents the breakdown of animals tested.

Results

\section{ESLH-Evoked Eating}

A significant difference in the incidence of ESLH-evoked eating was found between the Hi and Lo lines of the LC2 strain. Whereas $41.7 \%$

TABLE 1

Number of Animals Tested for ESLH or TailPinch-Evoked Eating in Experiment 1

\begin{tabular}{lcc}
\hline $\begin{array}{l}\text { Strain, line, } \\
\text { and sex }\end{array}$ & ESLH & $\begin{array}{r}\text { Tail } \\
\text { pinch }\end{array}$ \\
\hline $\begin{array}{l}\text { LC1 Lo } \\
\text { Female }\end{array}$ & 10 & \\
Male & 13 & 30 \\
LC1 Hi & & 30 \\
Female & 11 & 30 \\
Male & 11 & 30 \\
LC2 Lo & & \\
Female & 8 & 30 \\
Male & 10 & 30 \\
LC2 Hi & & \\
Female & 12 & 30 \\
Male & 12 & 30 \\
\hline
\end{tabular}


(10 of 24) animals of the Hi line ate in response to hypothalamic stimulation, only $5.5 \%$ ( 1 of 18 ) animals from the Lo line exhibited this behavior. In contrast, no difference between the $\mathrm{Hi}$ and Lo lines was found in the LC1 strain where $13.6 \%$ ( 3 of 22) and 13.0\% ( 3 of 23) of the animals showed ESLH-evoked eating, respectively. There was no significant difference in the current intensity required to evoke eating between the positive animals of both strains. In general, the LC1 strain tended to have a lower percentage of animals exhibiting ESLH eating than did the LC2 animals (13.3 vs $26.2 \%$ ) but this difference was not statistically significant. There were no statistically significant differences between the sexes of either strain, although it may be worth noting that the LC2 Hi females had the highest percentage (50\%) of animals exhibiting ESLH-evoked eating. The next highest success rate was $33.3 \%$ achieved by the LC2 Hi males.

\section{Tail-Pinch-Evoked Eating}

As described above, each animal received a tail-pinch score between 0 and 4 , depending on the number of the four tests in which it ate. A significant difference in tail-pinch-evoked eating score was found between the Hi and Lo lines of both the LC1 and LC2 strains (Mann-Whitney $U$ test corrected for ties). In contrast to the results in the ESLH-evoked eating tests, however, both the LC1 and LC2 Lo lines achieved a higher tail-pinch eating score than their respective Hi lines. The LC1 Lo line had an average score of 1.77 in contrast to 0.97 for the LC1 Hi line, while the LC2 Lo line had an average score of 1.45 compared to 0.97 for the LC2 Hi line. This difference between the Hi and Lo lines was also reflected in the percentage of animals that did not exhibit any tailpinch-induced eating during the four tests. Although some previous investigators have reported almost $100 \%$ success in producing tail-pinch "behaviors" (Antelman \& Szechtman, 1975), our success rate was relatively lower, probably because we gave each animal only four trials and counted as positive only instances where food was clearly eaten. In the LC1 strain, 57 and $33 \%$ of the animals in the Hi and Lo lines, respectively, did not eat during the tail-pinch tests, while the comparable scores were 53 and $32 \%$ for the Hi and Lo LC2 strain animals. Thus, the tendency for an inverse relationship between ESLH- and tail-pinch-evoked eating seemed to be a consistent phenomenon. The strains and lines that exhibited the lowest percentage of eating on the ESLH test had the highest average scores on the tail-pinch test. There were, however, no significant differences between the sexes in tail-pinch eating scores.

\section{EXPERIMENT 2}

In view of the similarities between the behaviors evoked by ESLH and tail pinch, the finding that the animal populations that scored lowest 
on one measure tended to score highest on the other measure was surprising. To determine whether this relationship could be generalized to other populations with more random genetic combinations than the selected lines, we studied the readiness to display tail-pinch eating by male rats from two other strains that were known to differ in ESLH-evoked eating. As already noted, Mittleman and Valenstein (1981) have reported that L-E rats exhibit significantly more ESLH-evoked eating than do S-D rats during tests identical to those used in Experiment 1.

We tested $20 \mathrm{~L}-\mathrm{E}$ and $24 \mathrm{~S}-\mathrm{D}$ male rats using the same tail-pinch procedure employed in Experiment 1. Animals were tested, however, over 10 daily 2-min tests instead of the four that were used for the LC1 and $\mathrm{LC} 2$ rats. $^{2}$ In addition to scoring whether animals ate during each test, we also recorded the amount of time the animals spent eating and biting or pulling the paper clip. Finally, as some measure of emotionality during the tail-pinch tests, we counted the number of fecal boli deposited by 18 rats (10 S-D and $8 \mathrm{~L}-\mathrm{E})$ during each 2 -min test.

\section{Results}

Although 10 daily tests were given to all animals, to make the results comparable with those obtained in Experiment 1 each animal was given a tail-pinch score (0-4) based on its test performance during the first four tests. The $20 \mathrm{~L}-\mathrm{E}$ rats had an average tail-pinch score of 0.52 , while the 24 S-D rats averaged 2.78 on the first four tests. Thus, the inverse relationship between tail-pinch- and ESLH-evoked eating suggested by testing the $\mathrm{LC} 1$ and $\mathrm{LC} 2$ populations was confirmed with the $\mathrm{L}-\mathrm{E}$ and S-D strains.

The difference in tail-pinch eating between the L-E and S-D rats was maintained over the 10 tests. The average total duration of eating for the 10 tests was $108.8 \mathrm{sec}$ for the L-E rats compared to $268.7 \mathrm{sec}$ for the S-D rats. This difference was statistically significant. The L-E rats spent significantly more time mouthing and biting the paper clip than did the $\mathrm{S}-\mathrm{D}$ animals $(\mathrm{L}-\mathrm{E}$ mean $=617.0 \mathrm{sec} ; \mathrm{S}-\mathrm{D}$ mean $=461.8 \mathrm{sec}$; average total for the 10 tests) and in general were judged to be more emotional as they vocalized more frequently during the tests. Higher emotionality of the L-E rats was also reflected in a significantly higher number of fecal boli deposited during the tail-pinch test (L-E mean = 28.2; S-D mean $=15.2$; average total for the 10 tests).

\section{DISCUSSION}

In view of what have been described as striking similarities between the behaviors evoked by ESLH and tail pinch, it was surprising to find that in both experiments, with the exception of the LC1 Hi line, the

\footnotetext{
${ }^{2}$ The assistance of Mark Litchman in testing animals in Experiment 2 is gratefully acknowledged.
} 
populations with the lower percentage of animals exhibiting ESLHevoked eating displayed the greater readiness to exhibit tail-pinch-induced eating. The fact that this inverse relationship was observed across populations with presumably different genetic combinations makes this finding quite robust. At present, we are attempting to explain this inverse relationship by considering (1) factors that either inhibit or facilitate eating; (2) differences in the quality and intensity of stimulation between ESLH and tail pinch; and (3) differences in emotionality between the populations of animals studied.

In considering factors that might inhibit or facilitate eating, we have assumed it to be well established that very high levels of emotionality interfere with eating because they are associated with competing behaviors such as very rapid locomotion or escape-directed responses and sometimes with an immobility or "freezing" response. In contrast, moderate levels of activation may facilitate eating at least under environmental and physiological conditions that normally predispose an animal to eat. There are numerous reports, for example, of mildly arousing stimuli, such as the presence of other animals, increasing food consumption in different species (Zajonc, 1965; Katz, 1953, pp. 117-118). It is reasonable to expect, therefore, that a high activation level generally interferes with eating while moderate levels may facilitate eating.

The ESLH and tail-pinch stimulation used here differed widely in the amount of activation they induced in the experimental animals. At the intensities generally used to evoke eating, ESLH tends to produce a moderate level of activation most commonly expressed as a forwardlocomoting exploratory pattern (Christopher \& Butter, 1968). Higher intensities of ESLH stimulation can produce very rapid locomotion and escape behaviors, but these almost never evoke eating. In contrast, tailpinch stimulation seems to become an intense, although not necessarily a painful, stimulus very rapidly for many animals and it commonly produces very rapid locomotion, circling, and escape behavior usually directed toward the part of the tail being pinched.

The amount of activation produced by any stimulus depends not only on its physical properties, but also on the emotionality or responsiveness of the animal receiving the stimulation. The L-E strain of rats used in Experiment 2, for example, appears to be generally more emotional and reactive than the $S-D$ animals. We have consistently observed that when they are not handled regularly, the L-E rats are more aggressive, startle easily, and resist being picked up. In response to tail pinch, the L-E rats vocalized and defecated more than the S-D animals and spent a greater amount of time attempting to remove the clip.

While similar observations were not systematically made for the $\mathrm{Hi}$ and Lo lines of the LC1 and LC2 strains, there is other evidence that the LC2 animals and particularly the LC2 $\mathrm{Hi}$ line respond more emo- 
tionally in several different test situations. Ganchrow et al. (1981) have shown that the LC2 Hi rats respond much more avidly to sweet-tasting substances than do either the LC1 Lo or Hi lines, although they do not differ in their rejection of a bitter-tasting substance. Also, Lieblich et al. (1980) have shown significant differences between 65-day-old LC1 and $\mathrm{LC} 2$ rats in the intensity and duration of the emotional changes produced by ablation of either the ventro-medial hypothalamic nuclei or the septal area. Following the ablations, the LC2 rats show greater rage and startle response than the LC1 animals, and within each of the two strains the Hi lines are characterized by a significantly greater emotional response than the Lo lines. When considered together with King's (1959) report that more emotional strains of rats exhibit greater rage and startle responses following septal lesions, it seems reasonable to conclude that the LC2 rats are more emotional than the LC1 rats and within the strains the Hi line rats are more emotional than those from the Lo lines. Consistent with that conclusion is the observation that the LC2 rats exhibit a greater response to chronic paresthesia (altered sensations) than LC1 rats (Inbal, Devor, Tuchendler, \& Lieblich, 1980). Following transection of the sciatic and saphenous nerves, the LC2 animals engaged in autotomy (self-mutilation) frequently, whereas the incidence was very low in $\mathrm{LC} 1$ animals.

Thus, as typically administered, it appears that tail pinch provides more intense stimulation than ESLH. Moreover, the populations of animals that do not readily exhibit tail-pinch eating are the relatively more emotional animals. If the conclusion that high levels of emotionality generally interfere with eating while moderate levels may facilitate eating is correct, then an inverse relationship between tail-pinch- and ESLHevoked eating becomes understandable. The more emotional populations of animals are less likely to exhibit eating during the more intense tailpinch stimulation, while the less intense hypothalamic stimulation is more likely to induce an optimal level of activation to facilitate the elicitation of eating. For the less emotional animals, tail pinch might not as readily evoke competing behaviors, whereas ESLH could provide less than optimal stimulation.

Although the hypothesis we have advanced to explain the inverse relationship between tail-pinch- and ESLH-evoked eating may be logical, we readily admit that more evidence is necessary to make the argument compelling. For example, we should identify strains with very high incidence of tail-pinch-induced eating and then test them for ESLH eating to ensure that the relation between the two is indeed negatively monotonic. An argument relying upon emotionality as a construct would also be strengthened by comparing strains that show a very great difference in emotionality such as the Maudsley reactive ("stress susceptible") and nonreactive ("stress resistant") strains. It would also be useful to test 
predictions that follow from the above argument. For example, animals from more emotional strains would be expected to show a greater readiness to eat in response to tail pinch when treated with a tranquilizing drug. It might also be predicted that more emotional strains would show a lower readiness to eat in response to ESLH when under the influence of a stimulating drug. One of these predictions might have already been confirmed, as Robbins, Phillips, and Sahakian (1977) have found that the minor tranquilizer chlordiazepoxide increases tail-pinch-induced eating in at least one strain of rats. It might be interesting to confirm these results with a barbiturate that is not confounded by the appetite-stimulating effects of some of the minor tranquilizers. Also, it should be expected that tail-pinch-elicited eating is possible in emotional animals at lower pressure levels than the relatively high level typically described in the literature as well as in the present study. Perhaps inconsistent with our hypothesis are the facts that the LC1 Hi line which showed low ESLH-induced eating also exhibited low tail-pinch-induced eating and the lack of sexual dimorphism in tail-pinch-induced eating which should have mirrored the tendency for such a dimorphism in ESLHinduced eating. However, the above mentioned predictions, deriving from the theory suggested to explain the obtained negative relation between eating evoked by ESLH and tail pinch, will provide an opportunity to decide whether the observed inconsistencies are substantial or due to sampling errors.

Finally, we should comment briefly on the numerous reports that ESLH- and tail-pinch-evoked eating as well as reward (Wise, 1980) are mediated by dopaminergic transmission. Recently, there have been several reports which have questioned the behavioral and neurochemical specificity of that evidence (Ettenberg, Koob, \& Bloom, 1981; Rowland, Marques, \& Fisher, 1980). The inverse relationship that we have reported here could also be viewed as evidence against any simple, one-transmitter theory of mediation; but the possibility that different dopaminergic pathways may be involved in the different behaviors or that a different level of dopamine activity might be optimal makes it premature to address this issue very seriously at this time.

\section{REFERENCES}

Antelman, S. M., \& Szechtman, H. (1975). Tail pinch induces eating in sated rats which appears to depend on nigrostriatal dopamine. Science, 189, 731-733.

Antelman, S. M., Szechtman, H., Chin, P., \& Fisher, A. E. (1975). Tail pinch-induced eating, gnawing and licking behavior in rats: Dependence on nigro-striatal dopamine system. Brain Research, 99, 319-337.

Antelman, S. M., Rowland, N. E., \& Fisher, A. E. (1976). Stimulation bound ingestive behavior: A view from the tail. Physiology and Behavior, 17, 743-748. (a)

Antelman, S. M., Rowland, N. E., \& Fisher, A. E. (1976). Stress related recovery from lateral hypothalamic aphagia. Brain Research, 102, 346-350. (b) 
Bachus, S. E., \& Valenstein, E. S. (1979). Individual behavioral responses to hypothalamic stimulation persist despite destruction of tissue surrounding electrode tip. Physiology and Behavior, 23, 421-426.

Barfield, R. J., \& Sachs, B. D. (1968). Sexual behavior: Stimulation by painful electric shock to the skin in male rats. Science, 161, 392-394.

Caggiula, A. R. (1972). Shock-elicited copulation and aggression in male rats. Journal of Comparative and Physiological Psychology, 83, 393-407.

Caggiula, A. R., \& Eibergen, R. (1969). Copulation of virgin male rats evoked by painful peripheral stimulation. Journal of Comparative and Physiological Psychology, 69, 414-419.

Christopher, M., \& Butter, C. M. (1968). Consummatory behaviors and locomotor exploration evoked from self-stimulation sites in rats. Journal of Comparative and Physiological Psychology, 66, 335-339.

Cohen, E., \& Lieblich, I. (1981). Sex differences in lateral hypothalamic self-stimulation. Behavioral and Neural Biology, 32, 448-451.

Coons, E. E., Levak, M., \& Miller, N. E. (1965). Lateral hypothalamus: Learning of food seeking response motivated by electrical stimulation. Science, 150, 1320-1321.

Ettenberg, A., Koob, G. F., \& Bloom, F. E. (1981). Response artifact in the measurement of neuroleptic-induced anhedonia. Science, 213, 357-359.

Ganchrow, J., Lieblich, I., \& Cohen, E. (1981). Consummatory responses to taste stimuli in rats selected for high and low rates of self-stimulation, Physiology and Behavior, 27, 971-976.

Greenspon, J. M., \& Fass, B. (1981). A reexamination of tail-pinch elicited eating following lateral hypothalamic lesions. Physiology and Behavior, 27, 487-492.

Harrell, L. E., Raubeson, R., \& Balagura, S. (1974). Acceleration of functional recovery following lateral hypothalamic damage by means of electrical stimulation in the lesioned area. Physiology and Behavior, 12, 897-899.

Inbal, R., Devor, M., Tuchendler, O., \& Lieblich, I. (1980). Autotomy following nerve injury: Genetic factors in the development of chronic pain. Pain, 9, 327-337.

Katz, D. (1953). Animals and Man. Harmondworth, England: Penguin.

King, F. A. (1959). Relationship of the "septal syndrome" to genetic differences in emotionality in the rat. Psychological Reports, 5, 11-17.

Koob, G. F., Fray, P. J., \& Iversen, S. D. (1976). Tail-pinch stimulation: Sufficient motivation for learning. Science, 194, 637-639.

Lieblich, I., Cohen, E., \& Beiles, A. (1978). Selection for high and for low rates of selfstimulation in rats. Physiology and Behavior, 21, 843-849.

Lieblich, I., Cohen, E., Marom, B., \& Dymshitz, J. (1980). A genetically mediated relationship between the readiness to self stimulate lateral hypothalamus and the intensity of septal and ventromedial hypothalamic rage syndromes. Brain Research, 185, 253-263.

Marques, D. M., Fisher, A. E., Okrutny, M. S., \& Rowland, N. E. (1979). Tail pinch induced fluid ingestion: Interactions of taste and deprivation. Physiology and Behavior, 22, 37-41.

Mendelson, J. (1966). Role of hunger in T-maze learning for food by rats. Journal of Comparative and Physiological Psychology, 62, 341-349.

Mendelson, J. (1972). Ecological modification of brain stimulation effects. International Journal of Psychobiology, 2, 285-304.

Mittleman, G., \& Valenstein, E. S. (1981). Strain differences in eating and drinking evoked by electrical stimulation of the hypothalamus. Physiology and Behavior, 26, 371-378.

Mufson, E. J., Balagura, S., \& Riss, W. (1976). Tail pinch-induced arousal and stimulusbound behavior in rats with lateral hypothalamic lesions. Brain Behavior, 13, 154-164.

Panksepp, J. (1971). Aggression elicited by electrical stimulation of the hypothalamus in albino rats. Physiology and Behavior, 6, 321-329. 
Phillips, A. G., Cox, V. C., Kakolewski, J., \& Valenstein, E. S. (1969). Object-carrying by rats: An approach to the behavior produced by brain stimulation. Science, 166, 903-905.

Phillips, A. G., \& Fibiger, H. C. (1973). Deficits in stimulation-induced feeding after intraventricular administration of 6-hydroxydopamine in rats. Behavioral Biology, 9, $749-754$.

Phillips, A. G., \& Fibiger, H. C. (1976). Long-term deficits in stimulation-induced behaviors and self-stimulation after 6-hydroxydopamine administration in rats. Behavioral Biology, 16, 127-143.

Phillips, A. G., \& Nikaido, R. S. (1975). Disruption of brain stimulation-induced feeding by dopamine receptor blockade. Nature (London), 258, 750-751.

Roberts, W. W., \& Carey, R. J. (1965). Rewarding effect of performance of gnawing aroused by hypothalamic stimulation in the rat. Journal of Comparative and Physiological Psychology, 59, 317-324.

Robbins, T. W., Phillips, A. G., \& Sahakian, B. J. (1977). Effects of chlordiazepoxide on tail pinch-induced eating in rats. Pharmacology, Biochemistry and Behavior, 6, 297-302.

Rowland, N. E., \& Antelman, S. M. (1975). Tail pinch induced eating parallels ingestion elicited by electrical brain stimulation. Federal Proceedings, 34, 373.

Rowland, N. E., \& Antelman, S. M. (1976). Stress-induced hyperphagia and obesity in rats: A possible model for understanding human obesity. Science, 191, 310-311.

Rowland, N., Marques, D. L., \& Fisher, A. E. (1980). Comparison of the effects of braindopamine depleting lesions upon oral behaviors elicited by tail pinch and electrical brain stimulation. Physiology and Behavior, 24, 273-281.

Sahakian, B. J., \& Robbins, T. W. (1977). Isolation-rearing enhances tail pinch-induced oral behavior in rats. Physiology and Behavior, 18, 53-58.

Steinbaum, E. A., \& Miller, N. E. (1965). Obesity from eating elicited by daily stimulation of hypothalamus. American Journal of Physiology, 208, 1-5.

Szechtman, H., Siegel, H. I., Rosenblatt, J. S., \& Komisaruk, B. R. (1977). Tail-pinch facilitates onset of maternal behaviour in rats. Physiology and Behavior, 19, 807-899.

Valenstein, E. S. (1976). The interpretation of behavior evoked by brain stimulation. In A. Wauquier \& E. T. Rolls (Eds.), Brain Stimulation Reward, pp. 557-575. New York: American Elsevier.

Valenstein, E. S. (1977), Brain mechanisms of reinforcement. In S. Obrador \& J. G. Martin-Rodrigues (Eds.), Neurosurgical Treatment in Psychiatry, Pain, and Epilepsy, pp. 27-49. Baltimore: University Park Press.

Valenstein, E. S., Cox, V. C., \& Kakolewski, J. W. (1970). A reexamination of the role of the hypothalamus in motivation. Psychological Review, 77, 16-31.

Wise, R. A. (1971). Individual differences in effects of hypothalamic stimulation. The role of stimulation locus. Physiology and Behavior, 6, 569-572.

Wise, R. A. (1980). The dopamine synapse and the notion of "pleasure centers" in the brain. Trends in Neuroscience, 3, 91-95.

Wyrwicka, W., \& Doty, R. W. (1966). Feeding induced in cats by electrical stimulation of the brain stem. Experimental Brain Research, 160, 152-160.

Zajonc, R. B. (1965). Social facilitation. Science, 149, 269-274. 